\title{
Elucidation and Decisional Risk in a Multi-Criteria Decision based on a Choquet Integral Aggregation-A Cybernetic Framework
}

\author{
J. MONTMAIN ${ }^{\mathrm{a}, \mathrm{b}, *}$, G. MAURIS ${ }^{\mathrm{c}}$ and A. AKHARRAZ ${ }^{\mathrm{a}}$ \\ a Laboratoire de Gi énie Informatique et d' Ingénierie de la Production, EMA Site EERIE, Parc Scientifique \\ ${ }^{\mathrm{b}}$ Commissariat àl'Énergie Atomique, CEA-ValrhôBP 171, Bagnols-sur-Cèze Cedex, France \\ c Laboratoire of Informatique, Systemes, Traitement de I Information et de la Connaissance, LISTIC, Universite de \\ Savoie, BP806, Annecy, France
}

\begin{abstract}
The authors are developing multi-criteria Decision-making support systems (DMSS) for project teams in charge of selecting a technical solution among alternatives. They propose a cybernetic framework to emphasize the link between decision-making (DM) and knowledge management processes in such projects. These DMSSs rely on the tracking of the accompanying knowledge production of long-term decisional processes by a collective with many actors. Based on knowledge-production management, this paper explains how to design decisional risk evaluation, monitoring and control aids and traceability functions for strategic choices and logical argumentation. The DMSS is seen as a recommender system for the project manager. Each possible solution involved in the decision-making process (DMP) is evaluated by means of a set of criteria. The evaluation results from an interpretation of the knowledge items in terms of satisfaction scores of the solutions according to the considered criteria. Aggregating these partial scores provides a ranking of all the possible solutions by order of preference. As criteria are sometimes interacting, the aggregation has to be based on adapted operators, i.e. Choquet integrals. Evaluating possible solutions by the knowledge contained in the knowledge base (KB) opens the way to automating the argumentation of the project team's decisions: the argumentation principle underlying this approach is based naturally on coupling a knowledge dynamical management system (KDMS) with the DMSS. The DMSS also evaluates the decisional risk that reflects the eventuality of a wrong selection due to the insufficiency of available knowledge at a given time in order to adopt a reliable solution. Decisional risk assessment corresponds to sensitivity analyses. These analyses are then exploited to control the decisional risk in time: they enable to identify the crucial information points for which additional and deeper investigations would be of great interest to improve the stability of the selection in the future. The knowledge management of a collective project is represented as a control loop: the KDMS is the actuator, the risk accompanying the decision is the controlled variable and is strongly linked to the entropy of the KB managed by the KDMS. Each of the three phases - intelligence, design, choice - of the DMP is identified to a function of the control loop: actuator, process and regulator. This cybernetic framework for decision has its origin in knowledge management activities for a great-scale project- the EtLD ${ }^{1}$ project of the French Atomic Commission (CEA) that concerns the management of high-level long-life radioactive waste in France.
\end{abstract}

KEY WORDS: decision-making support system; multi-criteria analysis; knowledge engineering; argumentation;

elucidation; decisional risk; Choquet integral aggregation

\footnotetext{
*Correspondence to: LGI2P, EMA - Site EERIE, Parc Scientifique Georges Besse, 30035 Nîmes Cedex 1, France. E-mail: jacky.montmain@ema.fr

${ }^{1}$ EtLD means Entreposage de très Longue Durée. This project concerns the management of high-level long-life radioactive waste in France.
}

\section{DECISION-MAKING IN AN ORGANIZATIONAL CONTEXT}

A critical examination of actual decision-making (DM) shows that a decision is not a precise, clearly identifiable act. Decision-making is a process: it is constructed, negotiated and follows a sinuous path over time (Paschetta and Tsoukiàs, 2000; Roy, 2004). According to a first rational model often associated with operational research, the decision 
should be the result of a comparative selection among various possible solutions. The decision makers and their advisers should carefully assess the risks and probable outcomes of each option, weigh their advantages and drawbacks, and ultimately select the most cost-effective solution. In terms of rational calculus, this analysis postulates the existence of a single actor who acts according to a hierarchy of preferences ordered according to their utility. This attractive theoretical model completely ignores the organizational aspects that are often implicit in the decisionmaking process (DMP).

Let us consider the specifications regarding the organizational decision. They were initially introduced by the economist and Nobel prizewinner H.A. Simon (1991). The specific characteristics of a DMP are:

- Knowledge acquisition and processing are probably a more crucial problem to achieve the right decision than the search for an apparently optimum decision. Indeed, the available information is generally incomplete and/or contradictory. The decision maker does not have a comprehensive knowledge of the situation, hence the term 'bounded rationality' used by Simon (1991). The latter opens a major field of investigation and implies that the dynamics of the decision process cannot be ignored (Simon, 1991, 1997). The idea is to improve the way humans use their reasoning and information-processing capabilities through adequate information processing and reasoning procedures.

- The bounds on knowledge of facts and hypotheses are due to the constraints of the organization, which selects or favours certain scenarios according to its own interests. In this case, the aim of a decision-making support system (DMSS) is to support the traceability of strategic choices and logical argumentation and develop models and methodologies that are compatible with cognitive modes used by human beings when confronted to a complex situation. Learning and DM are two strongly linked activities of these cognitive processes.

- Finding an optimum solution is irrelevant when several actors with different cultures and interests and many evaluation criteria are involved.
Indeed, there is a multiplicity of different overlapping, superimposed and conflicting rationalities (Sfez, 1992). To the same decisional situation can be associated several interpretations and several satisfactory decisional strategies depending on the point of view and the interests of the actors involved. The notion of an optimum solution is generally unrealistic, whereas achieving a consensus becomes a crucial issue in group DM, that is why argumentation is a basic functionality for a relevant DMSS.

- Simultaneous information availability in the whole organization and in time is nearly impossible. Solving delegation and co-ordination problems lead to serious difficulties: that is why the different phases of the DMP are not presented as a linear sequence but as a process with multiple possible loops. Thus, intelligence (information), design (representation), choice (selection) and review (revision) necessarily overlap in a looped DM process unlike the sequential process outlined (Figure 1 upper part).

- Multi-criteria analysis is more realistic and clearer to the decision-maker and thus contributes to the understanding of the decisional situation. In Simon's bounded rationality model (Simon, 1997), the manager is naturally inclined to adopt a single-criterion approach. This does not reflect the complexity of reality and leads to the adoption of a satisfactory but non-optimum solution. Multi-criteria decisions can mitigate this restriction. Building a model explicitly based on several criteria reflects and formalizes a natural and intuitive reasoning mode when faced with DM consisting in separately analysing each consequence (Roy and Bouyssou, 1993). Different mathematical schemes are used for combining pieces of information in order to draw the decision (Slowinski, 1998).

To capture all these different characteristics in the model supporting our DMSS, our approach is based on coupling a knowledge dynamical management system (KDMS) favouring and controlling the dynamic cognitive processes of learning, negotiating and representing knowledge phases, with a collective multi-criteria DM tool. Whereas Simon's model is a descriptive and cognitive model 
(a)
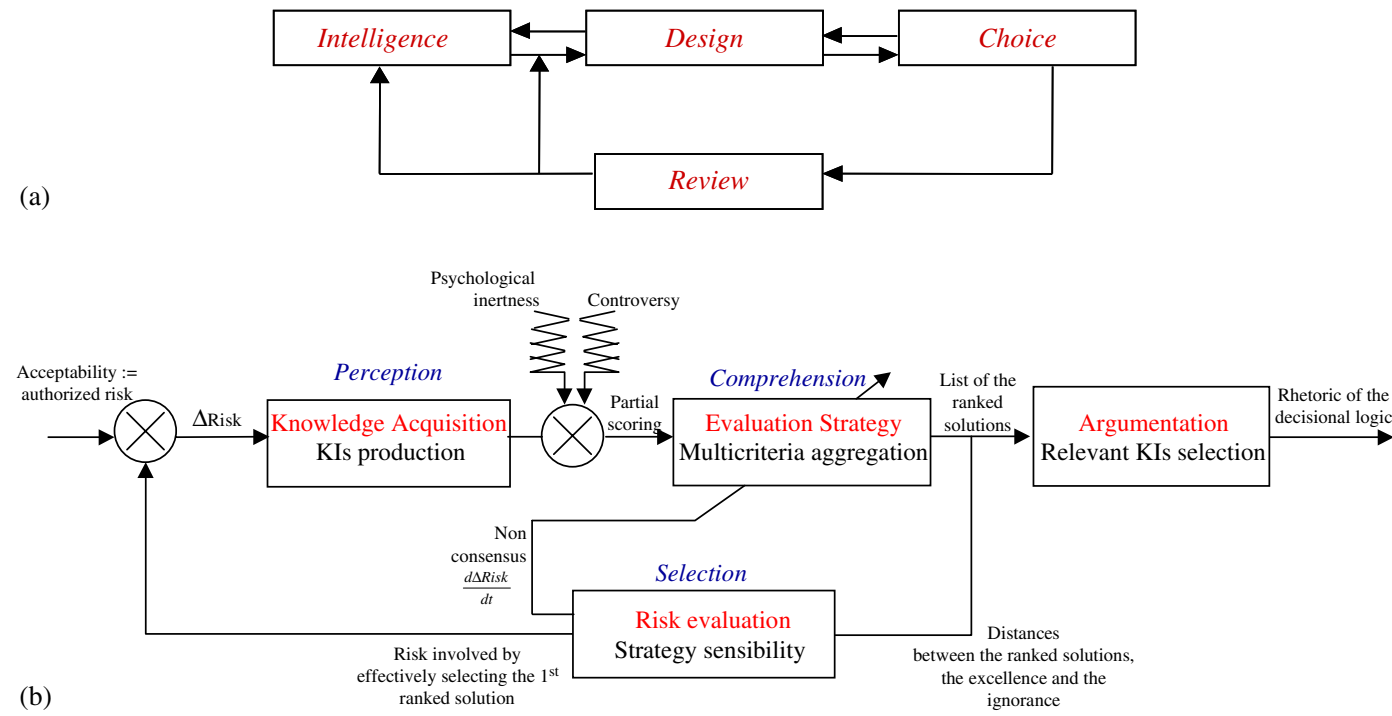

Figure 1. Process and risk control: (a) the cognitive model of Simon and (b) its cybernetic interpretation.

of the DMP, we implement a practical mathematical tool to aid DM in organization.

In summary, this paper explains how to transpose the previous specifications into a cybernetics modelling. This is achieved thanks to the notion of actionable knowledge that allows us to introduce control for directing the learning process for DM towards an expected issue. In particular, this induces controlled dynamics of the DMP. Section 2 provides the general outline of the control loop interpretation. Sections 3, 4 and 5, respectively, develop the intelligence-designchoice phases of Simon's model in terms of actuator, controlled process and regulator. The involved multi-criteria aggregation is based on the Choquet integral that allows taking mutual interactions between criteria into account. This multicriteria and cybernetic framework has its origin in the knowledge management of the EtLD project of the French Atomic Commission (CEA) which is described in Section 6. This project concerns the management of high-level long-life radioactive waste in France, and brings the specific functionalities of our DMSS - argumentation and decision sensitivity analyses - to light.

\section{A DMP CYBERNETIC FRAMEWORK}

Let us consider an organization getting involved in a project. Several technical or economical poten- tial solutions are possible in the project. The project team must thus develop a corpus of knowledge required to perform its tasks: evaluating the competing solutions and selecting the most relevant one. This corpus is the foundation on which collective processes of learning and DM can be built. Coupling a KDMS with a DMSS is natural: any decision must be based on facts, hypotheses, reflection, questioning, evidence, and accounts shared by the project collective and managed by the KDMS. The dynamics of the DMP is therefore necessarily founded on the knowledge corpus dynamics itself. The issue is then to control this learning phase.

The ability of a decision support system to justify a decision strategy is an expectation of project managers and working groups in order to establish presentation reports throughout the lifetime and on completion of the project (Dasarathy, 2000; Montmain et al., 2002; Moraïtis and Tsoukiàs, 2003; Niculae and French, 2003). As a consequence, the DMSS must be able to extract the most significant and decisive items of relevant knowledge from the associated KDMS. Justifying the evaluation or the ranking of the rival alternatives is a basic functionality of the KDMS-DMSS coupling. Decision elucidation is thus the first dimension of decision acceptability.

The second dimension to consider in the acceptability of a decision is the associated risk. When the potential solutions have been evaluated 
then the selection must be carried out. An error of judgement-i.e. selecting a solution that would later turn out to be a regrettable choice - is related to the amount of knowledge available to decide at that time. The epistemic uncertainty is related to the state of knowledge at the current time. At that time, the state of knowledge determines the reliability of the decision. When a small additional amount of new and relevant data appears sufficient to modify the ranking of the solutions, the epistemic uncertainty is high. To capture this concept of epistemic incertitude in a simple way we introduce the decisional risk that is related to the robustness of the solution ranking: the closer the competing solutions, the riskier the ranking and the higher the decisional risk. Thus, the decisional risk can be related to a distance between the alternatives. A formal definition will be later proposed in a multi-criteria evaluation framework (see Section 5).

Another way to explain the decisional risk concept is the following. It is merely introduced to capture the KB entropy ${ }^{2}$ at stake in the decision process: if the quantity of information (elementary knowledge items: KIs) necessary to modify the competing solution ranking is significant, the ranking is stable and the decisional risk is weak. If a minor quantity of information can modify the ranking then it is unstable, and the risk is high. The decisional risk is thus related to the reliability of the decision, and the reliability is a function of the information quantity, thus the decisional risk concept can be considered as an observer of the internal entropy of the KIs base of the KDMS.

Let us see how these notions find a place in a cybernetic interpretation of Simon's DMP representation (Figure 1 lower part). Let us consider a given knowledge corpus managed by a KDMS (based on an intranet server for example). Each item of knowledge (KI) managed by the KDMS can be seen as a judgement value on a given solution regarding a given evaluation criterion (knowledge acquisition box in Figure 1). The interpretation of a KI by its writer leads to a score reflecting the compatibility of the analysed solution with regard to a given criterion. Then a

\footnotetext{
${ }^{2}$ The notion of entropy is chosen here by analogy with the theory of information. But here, the entropy is a function of the rate of the potential decisions before and after the message delivery. It corresponds to the degree of indetermination in the communication.
}

strategy is modelled as a multi-criteria fusion to aggregate all the partial scores of a solution and assign an overall (aggregated) score to each solution (evaluation strategy box in Figure 1). Alternatives can thus be ranked w.r.t. their resulting aggregated score. The corresponding ranked list of considered solutions is achieved. This evaluation basically relies on the judgement values and thus the reverse must be achievable: elucidation of the ranking must be argued from the KI base (argumentation box in Figure 1). The decisional risk is then evaluated by the distances (based upon the differences of overall scores of the alternatives) $^{3}$ between the first-ranked solution and the other considered solutions (risk evaluation box in Figure 1). This risk is compared to a fixed value which defines the decision acceptability threshold. If the risk is higher than the acceptability threshold, an analysis of this risk factor indicates where there is a lack of KIs and where additional KIs should be the most relevant to reevaluate the solutions. The project manager can thus determine at each time which dimensions of the project are already decidable (there are enough relevant evidences in the current KIs base to reliably select an alternative) and which dimensions must be investigated deeper before a solution can be reliably identified. When the risk does not evolve anymore while it is still too high, it means that no consensus can be achieved with the current strategy: the strategy must be modified (model adaptation arrow in Figure 1).

As long as the entropy of the knowledge corpus of the KDMS evolves, it corresponds to a classical regulation process where the disturbances are the collective psychological inertness and controversy (disturbances arrows in Figure 1): these nonmeasurable disturbances do not correspond to required information flows identified by the risk analysis of the DMSS but to parasite information flows whose aim is either rejecting innovation or prejudicing interests.

This reasoning framework for an organizational process based on a prescriptive view of Simon's model is an analogy with a control theory representation (Figures 1(a) and (b)). The cognitive loop of Simon's model-the review phase-that completed his information-designchoice model is interpreted in terms of a feedback loop. From this viewpoint, the evaluation phase of

\footnotetext{
${ }^{3}$ Its computation is detailed in Section 5.2.
} 
the solutions is the process whose dynamics is to be controlled. The risk appears to be the controlled variable and the KDMS the actuator of the control loop. The elucidation that transcribes abstracted numerical evaluations for decision makers into rhetorical items in natural language can be assimilated to a kind of observation matrix that provides observable information of the process state. The acceptability threshold is seen as the setpoint of this control loop. The non-required flows of information are the non-measurable disturbances.

\section{THE INFORMATION PHASE-THE KDMS}

\subsection{Knowledge management}

Information technologies today facilitate communication and data exchange independently of the geographic location of the servers and actors involved. This technological performance is not sufficient, however, to allow the emergence of a true collective work mode in which all the cognitive processes-learning, argumentation, deliberation-assigned to a group of actors would reach or exceed a level of performance that could not be reached by each of the individuals alone (Penalva and Montmain, 2002; Montmain et al., 2002).

The group must first possess a shared memory that is the foundation on which collective processes of learning and DM can be built. The problem area we wish to address is that of dynamic knowledge-management systems (KDMS) (Penalva and Montmain, 2002). The dynamic structure of the KDMS promotes exchanges of 'professional' viewpoints, of value systems, interests or cultures that stimulate reactivity, deliberation and argumentation and can ultimately modify the dynamics of the project itself.

\subsection{The actuator: the KDMS}

A KDMS must capitalize on knowledge and skills, improve the visibility of the domain considered in the project (knowledge mapping) but also provide dynamic sharing of knowledge useful to the project. The neologism 'actionable knowledge' was introduced in organizational literature by Argyris and Schön (1978) to overcome the traditional distinction between knowledge and skill, i.e. the separation between epistemology (knowledge) and pragmatism (action). They pro- posed organizational models for action as early as 1974, and spoke of 'knowledge for action' in 1995. This work has shown that practitioners (knowing subjects engaged in action) best become aware of the action strategies they formulate - and can thus improve them - by reflecting on their actions, on their acquired knowledge. Transforming tacit knowledge into actionable knowledge is at the heart of the learning process. Thus, a KI is the basic element of the KDMS, an explicit statement of actionable knowledge, i.e. a piece of knowledge useful to the decision, which can be defined as any or all of the following:

- An informative data item considered to be useful.

- A basic interpreted knowledge element in the action project framework.

- A trace of reasoning.

- A sharable and reusable unit of meaning.

A decision is based not on raw information, but rather on the meaning of the information. An information item says nothing about what should be done: this can only be determined by interpretation-in other words, the meaning ascribed to it by a person in a given context. Through KIs, the interpreted basic knowledge leads us to distinguish between the informative value and the usefulness of a knowledge item within the project context. The KI characteristics are the following:

- Two KB indexes that correspond to the knowledge mapping and, respectively, define the competing solution and the problem type of the project the $\mathrm{KI}$ is related to (KB structure) (Figure 2(a)).

- The raw information that is discussed in the KI (informative value).

- A comment in natural language, i.e. a minimal rhetorical element (the basic interpreted knowledge element in the action project framework, i.e. the actionable knowledge).

- A date, the instant the KI appears in the KDMSS.

An example of KI is given in the application part of the paper in Figure 8. The actionable knowledge, i.e. the knowledge useful for the project, is managed by the KDMS that can thus be considered as the actuator of our looped DMP. 


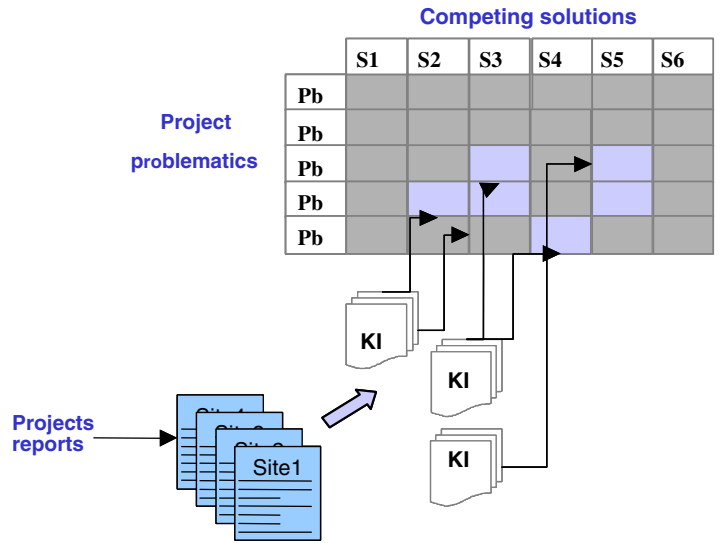

(a)

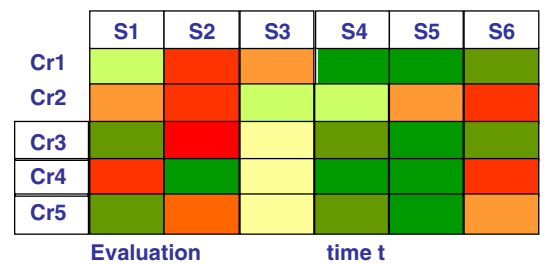

(b)

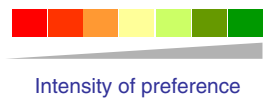

Figure 2. (a) Knowledge items mapping and (b) evaluation process by the KIs.

Learning collective knowledge and collective memory is the first cognitive process for which the KDMS offers a basis for a solution. In the following discussion it is explained how it also provides a natural medium for the other two cognitive processes related to collective intelligence: DM and explanation of the drawn decisions.

\section{THE DESIGN AND CHOICE PHASES-THE MULTI-CRITERIA EVALUATION PROCESS}

\subsection{The multi-criteria point of view}

On the basis of the KI mapping a project measurement space is defined: a grid evaluation (Figure 2(b)) of the competing solutions $\left(s^{k}\right)$ proposed over the life of the project according to a set of criteria $(\mathrm{Cr})$ is at the origin of the decision support functionalities. The project problematics of the mapping issue (Figure 2(a)) are derived by expertise into evaluation criteria for the evaluation issue (Figure 2(b)).

The basic idea of project evaluation refers to the collective choice theory, identifying the criteria as voters whose votes are the KIs and the project potential solutions as candidates for election. Indeed, since KIs are interpreted knowledge elements in the action project framework they correspond to judgement values about a given competing solution regarding a given criterion. An additional characteristic is thus attributed to the KI:

- A score (the judgement value) that captures the compatibility between a competing solution and the criterion of analysis (efficiency, cost, safety, etc.) the KI is related to. The score should be consistent with the comment in natural language, i.e. the minimal rhetorical element the KI conveys.

The score contained in the KI corresponds to a degree (between 0 and 1) of satisfaction of the criterion regarding the solution the KI is related to. This score is converted into a colour code ranging from non-satisfaction (red) to complete satisfaction (green), and which may take any intermediate value (Figure 2(b)) for man-machine interface reasons.

The scores of a KI assigned in the grid according to the KI indexes allow the differing intensities of voter preferences to be taken into account: each vote is an evaluation of a solution $i$ with respect to a criterion $j$. The overall score of a solution-its aggregated score - corresponds to the aggregation of partial scores obtained for each criterion, and can thus be equated with a criteria-driven election procedure. Consequently, the strategy of the organization is now supposed to be modelled by an aggregation operator that combines the partial scores on all the criteria as proposed in Section 4.2.2. The evaluation of the potential solutions is 
thus modelled by a three-level multi-criteria aggregation:

- Partial aggregation of the KIs assigned to a given box of the grid to evaluate the solution according to the criteria referred to in this box.

- Overall aggregation of the partial scores over a column with regard to a given strategy modelled by the aggregation operator.

- Implicit aggregation of KI judgement values in time with respect to the dynamics of the decisional process. This level of aggregation is not discussed in this paper because the temporal data fusion step not only depends on the previous partial aggregation of the KIs assigned to one box but it also strongly depends on the application.

Other observers of the changing perception of the DMP can complete the previous evaluation grid. The project itself is a dynamic organizational system; its state at $t_{i}$ can be explained by its previous states and new inputs. This allows to build a management chart or tracking grid corresponding to a table in which the rows are the proposed solutions, the columns symbolize the observation dates, and the items correspond to the overall scores of a solution at a given date $t_{i}$; the overall score is the result of aggregating the partial scores $x_{i}^{k}$ according to each criterion $i$ of solution $k$ in the evaluation grid at $t_{i}$ (Figure 3). The aggregation process is discussed in Section 4.2. Each column of this second grid thus represents a photo of the overall score of all the solutions at $t_{i}$. The evaluation grid at $t_{i}$ can thus be seen as a level of explanation of column $i$ of the management chart, i.e. the decision that would have been made - the solution that would have been adopted -if the project had been terminated on that date (Figure 3). The tracking grid shows the evolution of the perception of each solution as the project matures.

Another type of grid - the explanatory grid-'"' covering a given time interval can be used to track the partial scores of a given solution over time (Figure 3).

\subsection{Multi-criteria aggregation}

Having defined the framework and actors of the voting procedure, we must now specify the election mode or process, i.e. the manner in which the KI votes are composed and aggregated. Aggregation first concerns the combination of scores inherent in

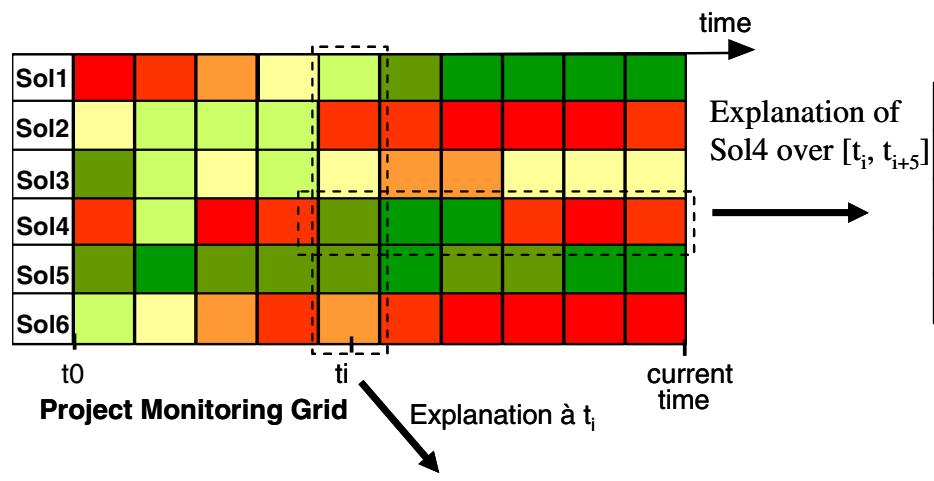

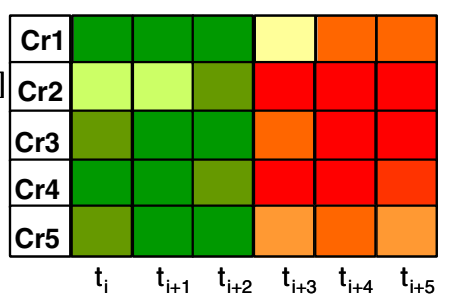

Evaluation of Sol4 over $\left[t_{i}, t_{i+5}\right]$

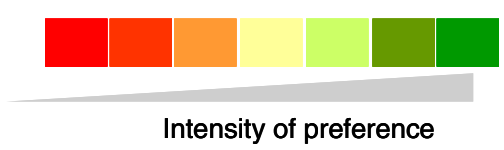

Evaluation at $\mathbf{t}_{\mathbf{i}}$

Figure 3. Evaluation grid, explanatory grid and tracking grid (management chart). 
the KIs relative to a given evaluation grid space. The overall score of a solution then requires that all the partial scores be aggregated according to each criterion. We will examine these points from the standpoint of multi-criteria aggregation.

4.2.1. Partial score of a competing solution with regard to a given criterion. The score conveyed by $\mathrm{KI}_{j}$ for solution $k$ with regard to criterion $i$ is denoted as $x_{i}^{k, \mathrm{KI}_{j}} \in[0,1]$ (it is the degree of satisfaction of solution $k\left(S^{k}\right)$, w.r.t. criterion $i$ expressed by $\left.\mathrm{KI}_{j}\right)$. The overall score $x_{i}^{k} \in[0,1]$ obtained by solution $k$ w.r.t. criterion $i$ results from the aggregation of the scores conveyed by all the knowledge items $\mathrm{KI}_{j}$; the $x_{i}^{k, \mathrm{KI}_{j}}$ terms refer to the evaluation grid space dealing with the evaluation of solution $k$ according to dimension $i$. Hence, an aggregation relation of the type $x_{i}^{k}=g\left(x_{i}^{k, \mathrm{KI}_{j}}\right)$, in which $g$ is an aggregation operator as proposed in Dubois (1983), Dubois and Prade (1984, 1985), Grabisch et al. (1998) and Marichal (1998).

However, at this step of aggregation, no strategic knowledge has to be used to define $g$ : this level of modelling does not reflect any strategic behaviour of the team project. Majority and unanimity rules, mean, median, ordered weighted average, limited veto, etc. can capture various possible behaviours (Yager, 1988; Zadeh, 1983; Kacprzyk, 1987; Koning, 1990). In the following, as this point is not the main concern of this paper, we simply choose the arithmetic mean for $g$ : $x_{i}^{k}=g\left(x_{i}^{k, \mathrm{KI}_{j}}\right)=1 / n b \mathrm{KI} \sum_{j=1}^{n b \mathrm{KI}} x_{i}^{k, \mathrm{KI}_{j}}$. All the KIs have the same relative importance, $x_{i}^{k}$ is a monotonic trade-off behaviour between good scores $x_{i}^{k, \mathrm{KI}_{j}}$ and bad scores $x_{i}^{k, \mathrm{KI}_{j^{\prime}}}$.

4.2.2. Global score of a competing solution. In return, this level of aggregation reflects a strategic behaviour. This aggregation is supposed to capture the strategy of the project team: priorities, importance and dependences between criteria.

Generally, the evaluation criteria are characterized by transverse as well as subordination relations. The hierarchical structure of the set of criteria is not unlike the hierarchical organization of specialist units or consultants that are expected to bring their experience and recommendations to the project manager's attention. Furthermore, coalitions or conflicts constitute interaction phenomena that may have an influence on the set of evaluation criteria. Transverse relations express co-ordination links that ensure the coherence of the scores at a given hierarchical level. Thus aggregation functions allowing to benefit from this semantic distinction in the relations among criteria (co-ordination and subordination relations) must be considered, for example, the Choquet integral (Grabisch and Roubens, 2000; Berrah et al., 2000). This operator is a good illustration of the aggregation of scores related by subordination and co-ordination dependences: in particular, it allows modelling of the relative importance of a criterion and its interaction with the other evaluation criteria. The properties of the Choquet integral and its relations with conventional aggregation operators have been discussed in depth, and the reader is encouraged to refer in particular to Grabisch et al. $(1995,1998)$ and Grabisch (1996).

Hereafter, we recall briefly some useful notations and definition of the Choquet Integral $C_{\mu}$. Let $C$ be a finite set of criteria.

\section{Definition 1}

A (discrete) fuzzy measure on $C$ is a monotone set function $\mu: P(C) \rightarrow[0,1]$, satisfying $\mu(\phi)=0$, $\mu(C)=1$; monotonicity means that $A \subset B \subset C$ $\Rightarrow \mu(A) \leqslant \mu(B) \leqslant \mu(C)$. Where $\mu(A)$ is seen as the weight of importance of the subset of criteria $A$.

\section{Definition 2}

Let $\mu$ be a fuzzy measure on $C$. The (discrete) Choquet integral of $x=\left\{x_{1}, x_{2}, \ldots, x_{n}\right\}$ w.r.t $\mu$ is defined by Grabisch (1997):

$$
C_{\mu}\left(x_{1}, \ldots, x_{n}\right)=\sum_{i=1}^{n}\left(x_{(i)}-x_{(i-1)}\right) \cdot \mu\left(A_{(i)}\right)
$$

Where the subscript (.) indicates a permutation such that: $x_{(0)}=0, \quad 0 \leqslant x_{(1)} \leqslant \cdots \leqslant x_{(n)} \leqslant 1$, and $A_{(i)}=\left\{c_{(i)}, \ldots, c_{(n)}\right\}$.

Let us consider a simple example with three criteria, $c_{1}, c_{2}$ and $c_{3}$ as illustration. Let us use the notations: $\mu_{i}=\mu\left(\left\{c_{i}\right\}\right)$ (resp. $\mu_{i j}=\mu\left(\left\{c_{i}, c_{j}\right\}\right)$ represents the importance of criterion $c_{i}$ (resp. $\left.\left(c_{i}, c_{j}\right)\right), \ldots$, for example, $\mu_{1}=0.4 ; \mu_{2}=\mu_{3}=0.3$; $\mu_{23}=0.8, \mu_{123}=1$. Let us define a solution $s^{k}$ by its profile vector $x^{k}=\left(x_{1}^{k}, x_{2}^{k}, x_{3}^{k}\right)=(0.9,0.5,0.8)$. Then $\left(x_{(1)}=0.5\right) \leqslant\left(x_{(2)}=0.8\right) \leqslant\left(x_{(3)}=0.9\right)$, this order implies that: $A_{(1)}=\left\{c_{(1)}, c_{(2)}, c_{(3)}\right\}=\left\{c_{2}\right.$, $\left.c_{3}, c_{1}\right\}, \quad A_{(2)}=\left\{c_{3}, c_{1}\right\}$ and $A_{(3)}=\left\{c_{1}\right\}$. Thus $\mu\left(A_{(1)}\right)=1 ; \mu\left(A_{(2)}\right)=0.8 ; \mu\left(A_{(3)}\right)=0.4$. And using (1), we have: $C_{\mu}\left(x_{1}, x_{2}, x_{3}\right)=0.78$. 
Following this approach, a decisional problem involving $n$ criteria requires $2^{n}$ coefficients in $[0,1]$ in order to define the fuzzy measure $\mu$ on every subset of $P(C)$. Of course a decision maker is generally not able to provide this information. Several references to the identification problem are nevertheless available in the literature (Grabisch, 1996; Marichal and Roubens, 2000; Angilella et al., 2004). Although this paper does not deal with the fuzzy measure identification problem, we believe that the form (1) of the Choquet integral is neither easy to use nor to understand for decision practitioners. Thus, we will consider only a particular case of Choquet fuzzy integrals known as the 2-additive measure (interactions are considered only two by two) (Grabisch and Roubens, 2000; Grabisch, 1997). In this case, only $n(n+1) / 2$ coefficients are required to define this fuzzy measure; the Choquet integral can then be expressed in the interpretable form as follows:

$C_{\mu}(x)=\sum_{i=1}^{n} v_{i} \cdot x_{i}-\frac{1}{2} \sum_{i>j}\left|\left(x_{i}-x_{j}\right)\right| \cdot I_{i j}$

where $v_{i}-\frac{1}{2} \sum_{j \neq i}\left|I_{i j}\right| \geqslant 0$ and where $v_{i}$ 's are the Shapley indices, representing the overall importance of each criterion relative to all the others, with the property $\left(\sum_{i=1}^{n} v_{i}=1\right) ; I_{i j}$ represents the interactions between pairs of the criteria $\left(c_{i}, c_{j}\right)$ with values contained in the interval $[-1 ; 1]$; a value 1 represents a positive synergy between the two criteria, a value of -1 is indicative of negative synergy, and a null value means there is no interactivity.

This formula makes it easier to interpret the contribution of the positive synergies (all $I_{i j}>0$ ) or negative synergies (all $I_{i j}<0$ ) to the overall score. If the interaction coefficients are all positive (synergy), then $C_{\mu}(x)<\sum_{i=1}^{n} v_{i} \cdot x_{i}$ and the behaviour is conjunctive: for a significant interaction $I_{i j}$, even if the score according to criterion $i$ is excellent $\left(x_{i}\right.$ close to 1$)$, a poor score for $x_{j}$ (close to 0 ) will entail a significant decrease of the overall score: $-\frac{1}{2} \cdot\left|x_{i}-x_{j}\right| \cdot I_{i j} \approx-\frac{1}{2} \cdot I_{i j}$.

The coefficients $v_{i}$ and $I_{i j}$ being more natural to decision makers, identification methods have been proposed in Grabisch and Roubens (2000) and Grabisch (1997) in order to use the transformation relation with the conventional fuzzy measure representation. Other methods based on the identification of these coefficients from experimental data exist but it is another problem (see
Grabisch and Roubens, 2000; Grabisch, 1997), that is not considered in this article.

\subsection{Elucidation of the ranking of the alternatives}

Let us consider that the solution that has been selected is $s^{k}$ : it has been adopted following an aggregation strategy (relative importance weights and interactions between criteria) identified with the aggregation operator $C_{\mu}$. Thus, for all $l=$ $1, \ldots, p$ (where $p$ is the solution number), we have $C_{\mu}\left(s^{k}\right) \geqslant C_{\mu}\left(s^{l}\right)$. An explanation as to why $s^{k}$ has been selected is of great value for decision makers and users. The answer to the ' $w h y$ ' question can be quantitatively expressed in terms of the relative influence or dominance of particular criterion scores on the ultimate decision (Montmain et al., 2002). Indeed, we are interested in the partial contribution of a specific criterion score for a considered aggregated score. Our approach to determine the contributions of each score is to decompose the aggregated score as a sum of terms in which partial scores are factorized. This is possible because the Choquet integral has a linear expression in each simplex $H_{\sigma}=\{x \in[0,1] / 0 \leqslant$ $\left.x_{\sigma(1)}^{k} \leqslant \cdots \leqslant x_{\sigma(n)}^{k} \leqslant 1\right\}$ (Akharraz et al., 2002). Two kinds of elucidation are considered as developed in the following subsections. Absolute elucidation corresponds to the search of the arguments that support the validity of a solution, whereas relative argumentation is related to the arguments that have led to prefer one solution to another one. This distinction is the same as the one made by a business unit between its marketing and its benchmarking departments: in the first one, the aim is to explain the intrinsic performances of the company; in the second one, the idea is to justify the company performances w.r.t. its rivals.

4.3.1. Absolute elucidation. First, it may be relevant to identify the elements related to the value of $s^{k}$ : it is an absolute elucidation. It consists in decomposing the aggregated score in a ranked sum of criterion score contributions. Then, it is possible to parameterize the levels of details required for the elucidation., i.e. a 'one-word' justification, the main reason for this preference, detailed reasons, or an exhaustive- and even anecdotal-report.

For this, by (1) we have

$C_{\mu}\left(s^{k}\right)=\sum_{i=1}^{n}\left(x_{(i)}^{k}-x_{(i-1)}^{k}\right) \mu\left(A_{(i)}^{k}\right)$

let us note : $\mu_{(i)}^{k}=\mu\left(A_{(i)}^{k}\right)$, then 


$$
\begin{aligned}
C_{\mu}\left(s^{k}\right)= & \left(\mu_{(1)}^{k}-\mu_{(2)}^{k}\right) x_{(1)}^{k}+\cdots+\left(\mu_{(i)}^{k}-\mu_{(i+1)}^{k}\right) x_{(i)}^{k} \\
& +\cdots+\mu_{(n)}^{k} x_{(n)}^{k} \\
\Rightarrow & C_{\mu}\left(s^{k}\right)=\sum_{i=1}^{n} \Delta \mu_{(i)}^{k} \cdot x_{(i)}^{k}
\end{aligned}
$$

where $\mu_{(n+1)}^{k}=0$, and $\Delta \mu_{(i)}^{k}=\mu_{(i)}^{k}-\mu_{(i+1)}^{k}$.

Thus, in a given simplex $H_{\sigma}=\{x \in[0,1] / 0 \leqslant$ $\left.x_{\sigma(1)}^{k} \leqslant \cdots \leqslant x_{\sigma(n)}^{k} \leqslant 1\right\}$ the Choquet integral is a linear operator. We can then simply re-rank the terms in (4) so that $\Delta \mu_{(j)}^{k} x_{(j)}^{k} \geqslant \Delta \mu_{(j+1)}^{k} x_{(j+1)}^{k}$ $\forall j=1, \ldots, n-1$.

The absolute contributions of the scores related to the criterion $c_{(j)}, \Delta \mu_{(j)}^{k} x_{(j)}^{k}$ (this term is defined as the absolute potential of criterion $c_{(j)}$ ) can then be partitioned into classes relative to the orders of magnitude of the $\Delta \mu_{(j)}^{k} x_{(j)}^{k} / \Delta \mu_{(1)}^{k} x_{(1)}^{k}$ ratio (Akharraz et al., 2002). The closer this ratio is to 1 , the greater the contribution of the score of the criterion $c_{(j)}$, and the more $c_{(j)}$ represents an essential dimension in the DM (local interpretation of elucidation).

In the case of a 2-additive fuzzy measure, the expression $\Delta \mu_{(i)}^{k}$ in (4) becomes (Akharraz et al., 2002)

$$
\Delta \mu_{(i)}^{k}=v_{(i)}+\frac{1}{2} \sum_{j>i} I_{(i)(j)}-\frac{1}{2} \sum_{j<i} I_{(j)(i)}
$$

where $v_{(i)}$ is the relative importance of criterion $c_{(i)}$ and $I_{(i)(j)}$ is the interaction between criteria $c_{(i)}$ and $c_{(j)}$.

Let us pursue the preceding example, the importance and interactions between the three elementary criteria are the following: $v_{1}=0.40, v_{2}=0.30$, $v_{3}=0.30$ and $I_{12}=0, I_{13}=0, I_{23}=0.4$. Thus since in this case, the criterion $C_{1}$ is independent from the two others, there are only two different simplexes $H_{\sigma_{k}}$ for the Choquet integral expression, i.e. $H_{23}$ : $P_{2}<P_{3}$ with $\Delta \mu_{1}=0.4, \Delta \mu_{2}=0.5, \Delta \mu_{3}=0.1$, and $H_{32}: P_{3}<P_{2}$ with $\Delta \mu_{1}=0.40, \Delta \mu_{2}=0.10, \Delta \mu_{3}=$ 0.50 (according to (2)). Then for a solution such that $s^{k}=\left(x_{1}^{k}=0.80, x_{2}^{k}=0.80, x_{3}^{k}=0.90\right)$, we obtain by Equation (4), $C_{\mu}\left(x_{1}, x_{2}, x_{3}\right)=0.81$. The absolute contributions are, respectively, $0.32,0.40$ and 0.09 . Thus, the higher contribution is the one relative to the criterion $\mathrm{C}_{2}$.

4.3.2. Relative elucidation. Another aspect of elucidation consists in providing evidence concerning the dimensions according to which $s^{k}$ was preferred over $s$, i.e. the dimensions for which $s^{k}$ made the difference over $s^{l}$. One simple issue is to use the following analysis:

$$
\forall l, \Delta C_{\mu R}\left(s^{k}, s^{l}\right)=C_{\mu}\left(s^{k}\right)-C_{\mu}\left(s^{l}\right)=\sum_{i=1}^{n} R_{(i)}^{k, l}
$$

where $R_{i}^{k, l}=\Delta \mu_{i}^{k} x_{i}^{k}-\Delta \mu_{i}^{l} x_{i}^{l}$.

This is a relative argument in which the quantities analysed are the sums of the individual relative potentials $R_{i}^{k, l}$. Note that many coefficients can be negative, though the global preferred solution has not necessarily the highest score on each criterion. We may adopt the same order-ofmagnitude reasoning for $\Delta C_{\mu R}\left(s^{k}, s^{l}\right)$ as for $C_{\mu}\left(s^{k}\right)$ as discussed in the preceding step, after using a permutation of the subscripts of $\Delta C_{\mu R}\left(s^{k}, s^{l}\right)$ for each solution $s^{k}$ to rank the individual relative potentials in decreasing order.

4.3.3. Rhetorical argumentation. At this stage of the relative and absolute argumentations, the most conclusive criteria for the decision are selected automatically. In other words, the evaluation grid spaces $(j, k)$ for which the associated partial scores play a decisive role in the final choices can be identified mechanically. Then, the last step is to extract from the KDMS the most characteristic KIs for the arguments we wish to assert (repeat the prior selection principle with the $x_{i}^{k, \mathrm{KI}_{j}}$, s and the arithmetic mean). The KIs, as rhetorical elements, provide the argumentation of the decision. Based on the KDMS, the elucidation algorithms enable to design traceability functions for strategic choices and logical argumentation. This function is the argumentation box in Figure 1(b). This can be considered as the observation matrix in terms of the process control theory.

\section{THE REVIEW PHASE-THE RISK REGULATOR}

\subsection{Decisional risk expression}

Once the partial scores on the database are evolving with time, it is important to consider the influence of a partial criterion score variation in the final ranking. Indeed, it is possible that a small amount of additional information is sufficient to modify the ranking of the solutions. The decisional risk can be seen as related to the reliability of the selection of a solution w.r.t. to informational disturbances (Section 2). Our risk notion is quite different from a conventional probabilistic one and is more in line with a sensitivity analysis of the solutions ranking 
that is itself linked to the sensitivity of the aggregated scores to partial score variations. We have preferred to associate the decisional risk concept to sensitivity analysis rather than to robustness analysis. We can say that robustness is generally connected to the fact that decision-aid methods often contain parameters whose values have to be chosen (more or less arbitrarily) by the user. Intuitively, a solution will be considered robust if the results obtained for different plausible values of the parameters' method do not contradict each other. In this line, several studies are available in the literature (Vincke, 1999a, 1999b; Roy, 1998). In our case, our sensitivity analysis is not concerned with the influence of the parameters on the decision result, but with the sensitivity of the aggregated score to any variations of the partial scores. These variations result from additional KIs in the KDMS, and they can be either disturbances or controlled variations (i.e. required or not required input changes).

The decisional risk matches the question "what is the risk choosing solution 1,1 st ranked, rather solution $k, k$ th ranked.' A decision is risky when a small amount of relevant information would be sufficient to reverse the ranking $s^{l} \geqslant s^{k}$. Thus, we propose to base the risk definition on a notion of distance $d\left(s^{l}, s^{k}\right)$ between the solution $s^{k}$ and the solution $s^{l}$ that has the highest global score. The risk expression $r$ varies in an opposite way versus this distance and thus can be defined by the following expression:

$r=1-\min _{k=2 \ldots p}\left(d\left(s^{l}, s^{k}\right)\right)$

where $p$ is the solution number and $d$ a normalized distance, e.g. the absolute value of the difference between the global scores associated to $s^{k}$ and $s^{l}$. Thus, $r$ varies between 0 (no risk because the best solution is far from the others and these will never catch up) and 1 (full risk because the best solution is close to the others). In other respects, the decisional risk expression gives information about the close solutions. Then, an important issue for the manager is the determination of the more sensitive evaluation dimensions: the decisional risk computation we propose meets this problem.

\subsection{Decisional risk computation}

The risk is associated to the sensitivity of the solution ranking. The lower the score increase of $s^{k}$ (to be at least equal to the score of $s^{l}$ ) is, the higher the risk is. To define the maximum risk, i.e. the reliability of the recommendation, a distance based on the $L_{1}$ norm between the solution $s^{l}$ and the others is introduced as in Equation (7). This distance is related to the optimization of the length (e.g. in the $L_{1}$ norm sense) of the path followed by any $s^{k}$ to reach the best solution $s^{l}$ : minimum length means maximum risk. Note that, for example, going from $s^{k}=\left(x_{1}^{k}=0.80, x_{2}^{k}=0.90, x_{3}^{k}=0.85\right)$ which has the overall score $C_{\mu}\left(s^{k}\right)=0.83$ to $s^{l}=$ $\left(x_{1}^{l}=0.90, x_{2}^{l}=0.95, x_{3}^{l}=0.90\right) \quad$ which has the overall score $C_{\mu}\left(s^{l}\right)=0.91$ could intuitively lead to increasing each $x_{i}^{k}$ up to $x_{i}^{l}$, i.e. $\left\|\delta^{k}\right\|_{1}=(0.90-$ $0.80)+(0.95-0.90)+(0.90-0.85)=0.20$. But as we will see below, this conventional behaviour is not the least lengthy one, i.e. a same overall score can be obtained with a smaller sum of the elementary score increases.

Indeed, the minimum increase $\boldsymbol{\delta}^{k}$ of the partial scores of $s^{k}$ such that $s^{l}$ achieves the same global evaluation with $C_{\mu}$ as $s^{l}$ is defined as the following optimization problem $(\mathrm{P})$ :

Objective function:

$\mid \min \|\delta\|_{L_{1}}$

Constraint:

$C_{\mu}\left(x_{1}^{k}+\delta_{1}^{k}, \ldots, x_{n}^{k}+\delta_{n}^{k}\right)=C_{\mu}\left(x_{1}^{l}, \ldots, x_{n}^{l}\right)$

Bound constraints:

$0 \leqslant \delta_{i}^{k} \leqslant 1-x_{i}^{k} \quad \forall i \in\{1, \ldots, n\}$

Let be $\overline{\boldsymbol{\delta}}^{k}=\left[\bar{\delta}_{1}^{k}, \ldots, \bar{\delta}_{p}^{k}\right]^{\mathrm{T}}$ a solution to $(\mathrm{P})$.

(P) is nonlinear because of the constraint $C_{\mu}\left(x_{1}^{k}+\delta_{1}^{k}, \ldots, x_{n}^{k}+\delta_{n}^{k}\right)=C_{\mu}\left(x_{1}^{l}, \ldots, x_{n}^{l}\right)$. This section provides a method to decompose the problem into $n$ ! linear programming problems where $C_{\mu}$ is linear $(n !$ is merely the theoretical algorithm complexity but can be efficiently reduced by simple heuristics as proposed below).

Let us now introduce the simplex $H_{\sigma}=\left\{x \in[0,1] / 0 \leqslant x_{\sigma(1)} \leqslant \cdots \leqslant x_{\sigma(n)} \leqslant 1\right\}, \quad$ where the Choquet integral has a linear expression (Equation (4)) with $\Delta \mu_{(i)}$ as linearity coefficient for the criterion $x_{(i)}$.

Let us consider the solution $s^{k}$ described by its score profile $\mathbf{x}^{k}=\left(x_{1}^{k}, \ldots, x_{n}^{k}\right)$ in the initial simplex $H_{\sigma_{0}}$ and corresponding to an overall score $C \mu\left(\mathbf{x}^{k}\right)$. The score to be reached is $C \mu\left(\mathbf{x}^{l}\right)$. The continuity and monotonicity ${ }^{4}$ of the Choquet integral ensure that a score profile $\mathbf{x} \in H_{\sigma_{0}}$ such that $C_{\mu}(\mathbf{x})=$

$\overline{{ }^{4} \text { Note that, } \forall H_{\sigma_{j}}},(1,1, \ldots, 1) \in H_{\sigma_{j}}$ and $C_{\mu}(1,1, \ldots, 1)$ 
$a \geqslant C_{\mu}\left(\mathbf{x}^{k}\right)$ necessarily exists for any value of $a$ in $\left[C_{\mu}\left(\mathbf{x}^{k}\right) ; 1\right]$ (i.e. $\mathbf{x}$ and $\mathbf{x}^{k}$ are comonotone).

Firstly, we are searching for this vector by increasing the scores of vector $\mathbf{x}^{k}$ in a minimum way in the $L_{1}$ norm sense. In this first step, since both score vectors are in the same simplex $H_{\sigma_{0}}$, the problem to be solved, $\left(P / H_{\sigma_{0}}\right)$, is thus a mere linear programming in $H_{\sigma_{0}}$ with

\section{Objective function:}

$\mid \min \left\|\boldsymbol{\delta}^{H_{\sigma_{0}}}\right\|_{L_{1}} \quad$ with $\boldsymbol{\delta}^{H_{\sigma_{0}}}=\left(\delta_{1}^{H_{\sigma_{0}}}, \ldots, \delta_{n}^{H_{\sigma_{0}}}\right)$

\section{Constraint:}

$\mid \sum_{q=1}^{n} \Delta \mu_{\sigma_{0}(q)} \cdot\left(x_{\sigma_{0}(q)}^{k}+\delta_{\sigma_{0}(q)}^{H_{\sigma_{0}}}\right)=C_{\mu}\left(\mathbf{x}^{l}\right)$

\section{Bound constraints:}

$\mid \begin{aligned} & 0 \leqslant \delta_{\sigma_{0}(q)}^{H_{\sigma_{0}}} \leqslant 1-x_{\sigma_{0}(q)}^{k} \quad \forall q \\ & x_{\sigma_{0}(q)}^{k}+\delta_{\sigma_{0}(q)}^{H_{\sigma_{0}}} \leqslant x_{\sigma_{0}(q+1)}^{k}+\delta_{\sigma_{0}(q+1)}^{H_{\sigma_{0}}} \quad \forall q^{5}\end{aligned}$

Let $^{5} \overline{\boldsymbol{\delta}}^{H_{\sigma_{0}}}=\left[\bar{\delta}_{1}^{H_{\sigma_{0}}}, \ldots, \bar{\delta}_{p}^{H_{\sigma_{0}}}\right]^{\mathrm{T}}$ a solution of $\left(P / H_{\sigma_{0}}\right)$. The last additional bound constraints of $\left(P / H_{\sigma_{0}}\right)$ enable to rewrite the Choquet Integral as a weighted sum and thus make the problem a linear one in a given simplex $H_{\sigma_{0}}$. In the following, we will see that the additional bound constraints enable to break down $(\mathrm{P})$ into several linear programming problems. Hence, we have found the minimal displacement $\overline{\boldsymbol{\delta}}^{H_{\sigma_{0}}}$ in $H_{\sigma_{0}}$ that satisfies $C_{\mu}\left(\mathbf{x}^{k}+\overline{\boldsymbol{\delta}}^{H_{\sigma_{0}}}\right)=C_{\mu}\left(\overline{\mathbf{x}}^{H_{\sigma_{0}}}\right)=C_{\mu}\left(\mathbf{x}^{l}\right)$. The computation of $\overline{\boldsymbol{\delta}}^{H_{\sigma_{0}}}$ is the initial step in the solving of (P).

Then, we have to consider the search of potential solutions to $(\mathrm{P})$ in all the simplices $H_{\sigma_{j}}$. Because of the properties of the Choquet integral mentioned above, we know: $\forall C_{\mu}\left(\mathbf{x}^{l}\right), \forall H_{\sigma_{j}}, \exists \mathbf{x}$ in $H_{\sigma_{j}}$ such that: $C_{\mu}(\mathbf{x})=C_{\mu}\left(\mathbf{x}^{l}\right)$. Let us denote $\boldsymbol{\delta}^{H_{\sigma_{j}}}$ as the displacement allowing to reach $C_{\mu}\left(\mathbf{x}^{l}\right)$ in $H_{\sigma_{j}}$ by going first from the scores vector $\mathbf{x}^{k}=\left(x_{1}^{k}, \ldots\right.$, $\left.x_{n}^{k}\right)$ in $H_{\sigma_{0}}$ to the border between $H_{\sigma_{0}}$ and $H_{\sigma_{j}}$ (the border point is denoted $\mathbf{x}^{T_{\sigma_{0} \sigma_{j}}}$ with $\overrightarrow{\mathbf{x}^{k} \mathbf{x}^{T_{\sigma_{0} \sigma_{j}}}}=\boldsymbol{\delta}^{T_{\sigma_{0} \sigma_{j}}}$ and $\forall \boldsymbol{\delta}_{i}^{T_{\sigma_{0} \sigma_{j}}}, \boldsymbol{\delta}_{i_{\sigma_{0} \sigma_{j}}}^{T_{\sigma_{0}}} \geqslant 0$ (decreasing is not considered) and then to $\mathbf{x}^{H_{\sigma_{j}}}$ in $H_{\sigma_{j}}$ such that $C_{\mu}\left(\mathbf{x}^{H_{\sigma_{j}}}\right)=C_{\mu}\left(\mathbf{x}^{l}\right)$ (the displacement from $\mathbf{x}^{T_{\sigma_{0} \sigma_{j}}}$ to $\mathbf{x}^{H_{\sigma_{j}}}$ is denoted $\left.\boldsymbol{\delta}^{H_{\sigma_{j}}}\right):\left\|\overrightarrow{\mathbf{x}^{k} \mathbf{x}^{H_{\sigma_{j}}}}\right\|_{L_{1}}=\left\|\overrightarrow{\mathbf{x}^{k} \mathbf{x}^{T_{\sigma_{0} \sigma_{j}}}}\right\|_{L_{1}}+\left\|\overrightarrow{\mathbf{x}^{T_{\sigma_{0} \sigma_{j}}} \mathbf{x}^{H_{\sigma_{j}}}}\right\|_{L_{1}}=$

\footnotetext{
${ }^{5}$ These constraints mean: increasing the elementary performances cannot change the partial score ranking in order to remain in the same simplex $H_{\sigma_{0}}$.
}

$\boldsymbol{\delta}^{T_{\sigma_{0} \sigma_{j}}}+\boldsymbol{\delta}^{H_{\sigma_{j}}}$ (this equality is due to the use of the $L_{1}$ norm and $\forall \delta_{i}^{T_{\sigma_{0} \sigma_{j}}}, \delta_{i}^{T_{\sigma_{0} \sigma_{j}}} \geqslant 0$ and $\forall \delta_{i}^{H_{\sigma_{j}}}, \delta_{i}^{H_{\sigma_{j}}} \geqslant 0$ because decreasing is not allowed).

We have $C_{\mu}\left(\mathbf{x}^{k}+\boldsymbol{\delta}_{i}^{T_{\sigma_{0} \sigma_{j}}}+\boldsymbol{\delta}^{H_{\sigma_{j}}}\right)=C_{\mu}\left(\mathbf{x}^{T_{\sigma_{0} \sigma_{j}}}+\right.$ $\left.\boldsymbol{\delta}^{H_{\sigma_{j}}}\right)=C_{\mu}\left(\mathbf{x}^{l}\right)$.

First, we have to determine the closest border point $\mathbf{x}^{T_{\sigma_{0} \sigma_{j}}}$ to $\mathbf{x}^{k}$, i.e. $\boldsymbol{\delta}^{T_{\sigma_{0} \sigma_{j}}}$ is minimum. Let us define the elementary positive translation $T(q, r)$ that consists to add $\delta_{q r}$ to the partial score $x_{q}$ of a solution $s$ in order to modify the initial ranking $x_{\sigma(1)} \leqslant \cdots \leqslant x_{q} \leqslant \cdots \leqslant$ $x_{r} \leqslant \cdots \leqslant x_{\sigma(n)}\left(\right.$ simplex $\left.H_{\sigma_{\sigma r}}\right)$ into $x_{\sigma(1)} \leqslant \cdots \leqslant x_{r} \leqslant$ $x_{q} \leqslant \cdots \leqslant x_{\sigma(n)}$ (simplex $\left.H_{\sigma_{r q}}\right)$. The minimum elementary displacement necessary to this ranking inversion is $\left(x_{r}^{i}-x_{q}^{i}\right)$, i.e. when $x_{r}^{i}=x_{q}^{i}$. The attainable point verifies $x_{\sigma(1)} \leqslant \cdots \leqslant x_{q}=x_{r} \leqslant \cdots$ $\leqslant x_{\sigma(n)}$ and belongs both to $H_{\sigma_{q r}}$ and $H_{\sigma_{r q}}$, hence it belongs to the border of $H_{\sigma_{q r}}$ and $H_{\sigma_{r q}}$.

More generally any ranking inversion of the partial scores is an application $T_{\sigma_{1} \sigma_{2}}$ defined by

$T_{\sigma_{1} \sigma_{2}} \mid \begin{gathered}H_{\sigma_{1}} \rightarrow H_{\sigma_{2}} \\ x^{H_{\sigma_{1}}} \rightarrow x^{H_{\sigma_{2}}}\end{gathered}$

where $T_{\sigma_{1} \sigma_{2}}$ is the composition of elementary translations (resulting from ranking permutations) as defined previously. It is thus a translation too. The way the elementary translation vectors (only border points $x^{\sigma_{2}}$ are processed) are built guarantees $\boldsymbol{\delta}^{T_{\sigma_{1} \sigma_{2}}}=\left\|\overrightarrow{\mathbf{x}^{H_{\sigma_{1}}} \mathbf{X}^{H_{\sigma_{2}}}}\right\|$ to be minimal.

$\boldsymbol{\delta}^{T_{\sigma_{0} \sigma_{j}}}$ is thus easily computed. Then, $\overline{\boldsymbol{\delta}}^{H_{\sigma_{j}}}$ is to be computed.

For each of the $(\mathrm{n} !-1)$ simplices $H_{\sigma_{j}}$, the minimal displacement $\overline{\boldsymbol{\delta}}^{H_{\sigma_{j}}}$ is computed by the following linear programming problem $\left(P / H_{\sigma_{j}}\right)$ :

Objective function:

$\mid \min \left\|\boldsymbol{\delta}^{H_{\sigma_{j}}}\right\|_{1} \quad$ with $\boldsymbol{\delta}^{H_{\sigma_{j}}}=\left(\delta_{1}^{H_{\sigma_{j}}}, \ldots, \delta_{n}^{H_{\sigma_{j}}}\right)$

\section{Constraint:}

$\mid \sum_{q=1}^{n} \Delta \mu_{\sigma_{j}(q)} \cdot\left(x_{\sigma_{j}(q)}^{T_{\sigma_{0} \sigma_{j}}}+\delta_{\sigma_{j}(q)}^{H_{\sigma_{j}}}\right)=C_{\mu}\left(\mathbf{x}^{l}\right)$

Bound constraints:

$\mid \begin{aligned} & 0 \leqslant \delta_{\sigma_{j}(q)}^{H_{\sigma_{j}}} \leqslant 1-x_{\sigma_{j}(q)}^{T_{\sigma_{0} \sigma_{j}}} \quad \forall q \\ & x_{\sigma_{j}(q)}^{T_{\sigma_{0} \sigma_{j}}}+\delta_{\sigma_{j}(q)}^{H_{\sigma_{j}}} \leqslant x_{\sigma_{j}(q+1)}^{T_{\sigma_{0} \sigma_{j}}}+\delta_{\sigma_{j}(q+1)}^{H_{\sigma_{j}}} \quad \forall q\end{aligned}$

The solution to $\left(P / H_{\sigma_{j}}\right)$ is $\overline{\boldsymbol{\delta}}^{H_{\sigma_{j}}}$, and $\overline{\mathbf{x}}^{H_{\sigma_{j}}}=\mathbf{x}^{T_{\sigma_{0} \sigma_{j}}}$ $+\overline{\boldsymbol{\delta}}^{H_{\sigma_{j}}}$

Then, we can compute

$\left\|\overrightarrow{\mathbf{x}^{k}} \overline{\mathbf{x}}^{H_{\sigma_{j}}}\right\|_{L_{1}}=\left\|\boldsymbol{\delta}^{T_{\sigma_{0} \sigma_{j}}}\right\|_{L_{1}}+\left\|\overline{\boldsymbol{\delta}}^{H_{\sigma_{j}}}\right\|_{L_{1}}$ 
Finally, we obtain the solution to (P)

$\overline{\boldsymbol{\delta}}^{k}=\min _{j}\left(\left\|\boldsymbol{\delta}^{T_{\sigma_{0} \sigma_{j}}}\right\|_{L_{1}}+\left\|\overline{\boldsymbol{\delta}}^{H_{\sigma_{j}}}\right\|_{L_{1}}\right)$

Note that, as soon as $\left\|\boldsymbol{\delta}^{T_{\sigma_{0} \sigma_{j}}}\right\|_{L_{1}}>\overline{\boldsymbol{\delta}}^{H_{\sigma_{0}}}$, search in $H_{\sigma_{j}}$ is no longer required. As a consequence, although the theoretical complexity of the algorithm is $n$ !, simple heuristics enable to reduce it significantly.

The decisional risk that characterizes the selection is then

$r=1-\min _{k=2 \ldots p} d\left(s^{l}, s^{k}\right) \quad$ with $d\left(s^{l}, s^{k}\right)=\frac{\overline{\boldsymbol{\delta}}^{k}}{n}$

Let us consider again the preceding example where $s^{l}=\left(x_{1}^{l}=0.90, x_{2}^{l}=0.95, x_{3}^{l}=0.90\right) \quad$ with the overall score $C_{\mu}\left(s^{l}\right)=0.91$ and $s^{k}=\left(x_{1}^{k}=0.80, x_{2}^{k}\right.$ $=0.90, x_{3}^{k}=0.85$ ) with the overall score $C_{\mu}$ $\left(s^{k}\right)=0.83$. Obviously, $s^{l}$ has to be preferred to $s^{k}$. According to the algorithm exposed before, the lower increase vector is $\overline{\boldsymbol{\delta}}^{k}=\left(\overline{\boldsymbol{\delta}}_{1}^{k}=0.1, \overline{\boldsymbol{\delta}}_{2}^{k}=\right.$ $\left.0.05, \overline{\boldsymbol{\delta}}_{3}^{k}=0.05\right)$. Thus we have $\overline{\boldsymbol{\delta}}^{k} / 3=0.11 / 3=$ 0.037 . Thus, the decisional risk is $1-0.037=0.0963$.

Note that by considering to increase each $x_{i}^{k}$ up to $x_{i}^{l}$ would have led to a decisional risk of $1-0.20$ / $3=0.933$.

\subsection{Decisional risk control}

At this stage of the project management issue, the risk is compared to a fixed acceptability threshold (Figure 1(b)). When it is below this threshold, the manager can consider $s^{l}$ as a reliable solution for his/her project: the quantity of information (the KIs) necessary to modify the current ranking would be considerable. $s^{l}$ is a stable or robust solution, the decisional risk is weak and can be legitimated (Section 4.3). When the risk is over the acceptability threshold, the situation is not decidable. It is moreover sensitive to any additional informational disturbances; the manager needs further KIs to reach a decision.

The following computation provides elements to determine the dimensions of the project for which additional KIs would be the most relevant. The necessary displacement to be made by $s^{k}$ to reach $s^{1}$ regarding criteria $j$ is $\bar{\delta}_{j}^{k}$ (see Equation (11)), this improvement induces a corresponding 'energy'. The latter is defined by:

$W_{j}^{l k}=(\sum_{H_{\sigma} \in H_{\sigma_{0}} \rightarrow H_{\sigma_{F}}}^{\text {(crossed hyperplans) }} \underbrace{\Delta \mu_{j}^{H_{\sigma}} \cdot \delta_{j}^{T_{\sigma \sigma^{\prime}}}}_{\text {Translation from } H_{\sigma} \text { to } H_{\sigma^{\prime}}}+\underbrace{\Delta \mu_{j}^{H_{\sigma_{F}}} \cdot \bar{\delta}_{j}^{H_{\sigma_{F}}}}_{\text {Simplex in } H_{\sigma_{F}}})$
$H_{\sigma_{F}}$ is the simplex in which the solution to $(\mathrm{P})$ has been found. The sum in (13) is defined over all the simplices crossed by the optimal path defined in the risk calculus algorithm. Each partial energy $W_{j}^{l k}$ thus results from the translation displacements and the ending simplex displacement w.r.t. criterion $j$ : it is locally expressed as the product of a local effort (local weight) $\Delta \mu_{j}^{H_{\sigma}}$ and an elementary displacement $\delta_{j}^{T_{\sigma^{\prime}}}$ or $\bar{\delta}_{j}^{H_{\sigma_{F}}}$ in the ending simplex.

The overall energy necessary to achieve $s^{k} \geqslant s^{l}$ is then

$W^{l k}=C_{\mu}\left(s^{l}\right)-C_{\mu}\left(s^{k}\right)=\sum_{j=1}^{n} W_{j}^{l k}$

From (11), using the mechanical analogy (the scalar product of a force and a displacement matches with an energy), the average effort $E_{1 k}^{j}$ to be made by $s^{k}$ to reach $s^{l}$ regarding criteria $j$ is defined by:

$$
\begin{aligned}
W_{j}^{l k} & =E_{j}^{l k} \cdot \bar{\delta}_{i}^{k} \Rightarrow E_{j}^{l k} \\
& =\frac{\left(\sum_{H_{\sigma} \in H_{\sigma_{0}} \rightarrow H_{\sigma_{F}}} \Delta \mu_{j}^{H_{\sigma}} \cdot \delta_{j}^{T_{\sigma \sigma^{\prime}}}+\Delta \mu_{j}^{H_{\sigma_{F}}} \cdot \bar{\delta}_{j}^{H_{\sigma_{F}}}\right)}{\left\|\overline{\boldsymbol{\delta}}^{k}\right\|_{L_{1}}}
\end{aligned}
$$

$E_{j}^{l k}$ - the effort-can be interpreted as the average weight of criteria $j$ along $H_{\sigma_{0}} \rightarrow H_{\sigma_{F}}$. The higher the $E_{j}^{l k}$, the more efficient the energy regarding criteria $j$. As a consequence, the manager's recommendation to the project team should be to bring first new and determinant results between $s^{k}$ and $s^{l}$ regarding the dimensions $j^{*}$ such that $E_{j^{*}}^{l k}$ $\geqslant(1-\varepsilon) \cdot \max _{j=1 \ldots n} E_{i}^{l k}$ ( $\varepsilon$ is a parameter to be determined w.r.t. the application) because they are the riskier dimensions of the current selection in the sense that they are more susceptible to reverse the current ranking. This recommendation thus provides the control information signal returned to the Information box (the actuator) of our control loop in Figure 1 (Akharraz et al., 2004a). But note that it is merely local and qualitative: the aim is not to establish a global control law over the whole trajectory.

\section{ORIGIN OF THIS WORK}

The coupling of our KDMS with a DMSS originated in the knowledge management of a large-scale project-the EtLD project of the 
French Atomic Commission (CEA) that concerns the management of high-level long-life radioactive waste in France. For obvious confidentiality reasons, the aim of this section is not to illustrate numerically the previous results but to illustrate how initial knowledge management needs have finally evolved into DM requirements. Numerical details of the algorithms can be found in applications with no political stake: film programming in a video-club (Akharraz et al., 2004b); management of a manufacturing organization (Montmain et al., 2004; Berrah et al., 2005); recommendation for e-business activities (Denguir-Rekik et al., 2006).

France does not have a long-term programme for managing fuel that is not designated for reprocessing. Since $\mathrm{EDF}^{6}$ does not plan to reprocess all its fuel, the question of the future of irradiated fuel needs to be addressed. A long-term solution is being studied in the framework of law no. 91-1381 of 30 December 1991 concerning research on the management of radioactive waste. This law directly concerns the management of 'high-activity, long-life waste'. That law requires the French Government to present to Parliament an annual report stating the progress of research and of work on evaluation and comparison of methods of packaging and of long-term surface storage of radioactive waste.

At the end of a 15 year period, the Government will send to Parliament an overall report evaluating this research, accompanied by a bill authorizing, if found appropriate, the creation of a storage centre for high-activity, long-life waste. The result of the $\mathrm{CEA}^{7}$ studies for the project are controlled by the National Evaluation Commission for research on the management of radioactive waste (CNE), instituted by law 91-1381.

The purpose of research is first to condition long-lived radioactive waste in order to ensure their safe and durable containment in the form of handle-able containers, and also to investigate long-term storage facilities, both ground and underground, in order to protect those containers' durably and to ensure their long-term retrievability.

The CEA has proposed a dozen 'families' of competing preliminary concepts or solutions for

\footnotetext{
$\overline{6}$ Electricité de France.

7 Commissariat à l'Energie Atomique, i.e. French Atomic
}

one or several EtLD. ${ }^{8}$ The Commission has given priority to studies on irradiated fuel. According to the $\mathrm{CNE}$, the researchers are still studying

- A design composed of a network of galleries buried between 30 and $50 \mathrm{~m}$ below the surface of the ground, for 'irradiated fuel, B and C waste'.

- A surface or half-buried installation.

- A concrete bunker, possibly half-buried, cooled by natural ventilation, like Cascad at Cadarache.

- A concrete bunker, possibly half-buried, providing centralized storage for material with strong thermal density and having an evolutionary system of cooling.

- A regional depository for irradiated fuel under shelter, modular and strictly surface. It would receive standardized containers of irradiated fuel.

All the potential solutions to EtLD are to be evaluated and compared w.r.t. the analysis perspectives of EtLD, submitted for approval by the CNE.

Initially, a CEA project manager was designated in 1997 for EtLD. More than 100 engineers were concerned with this project and the number of analyses relative to the EtLD studies exponentially increased. Considering the amount of documents he had to handle, the head manager first made up his mind to develop an intranet tool for document management. Thus, our laboratory was initially in charge of developing this electronic document management system. This first work phase led us to develop a KDMS named the referential knowledge base (RKB), a virtual collaborative platform whose objectives are (Penalva and Mountmain, 2002):

- Effective and rapid distribution of information and knowledge within the project teams.

- Providing an information source for external partners, especially for the industry in search of competence.

- Ensure synergetic results by avoiding parallel research and development within the project.

The virtual collaborative platform offers the management of common databases, common

\footnotetext{
${ }^{8}$ Entreposage de très Longue Durée, i.e. very long-term storage of waste.
} 
newsletters and provides a common Internet presence to distribute information and knowledge within the network. Technically, this is an intranet tool: a Web server interfaced with a SQL data base and a document base (Spirit) (Figure 4). Centralized administration is preferable from an organizational standpoint, although the system can be distributed among authorized agents.

The collection of documents and data widely distributed throughout the organization is generally a weighty and difficult task. The proposed solution overcomes this obstacle by eliminating the collection step altogether in favour of submissions by each actor. The intranet was operational in 1998 (Figure 5).

The need for structuring the documents base rapidly appeared: because the EtLD's main objective was to evaluate and compare different long-term storage possibilities, the base was structured in relation to the potential solution list. The solutions were subdivided into two main classes: surface storage (e.g. Cascade (France): interim storage in shafts) and subsurface storage (e.g. Clab (Sweden): underground pools). The evaluation problem of the potential solutions in EtLD was rather complex, which is why the

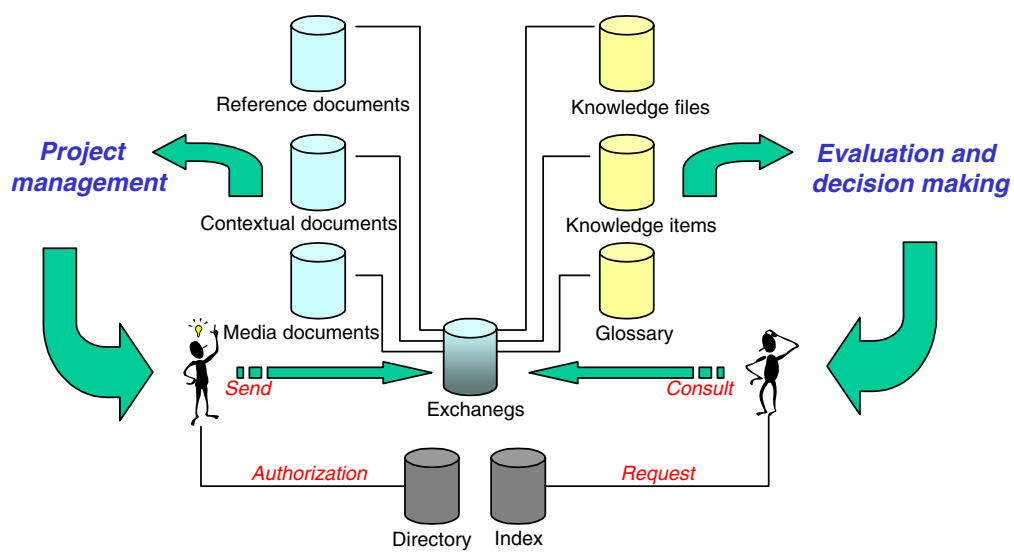

Figure 4. RKB base structure.

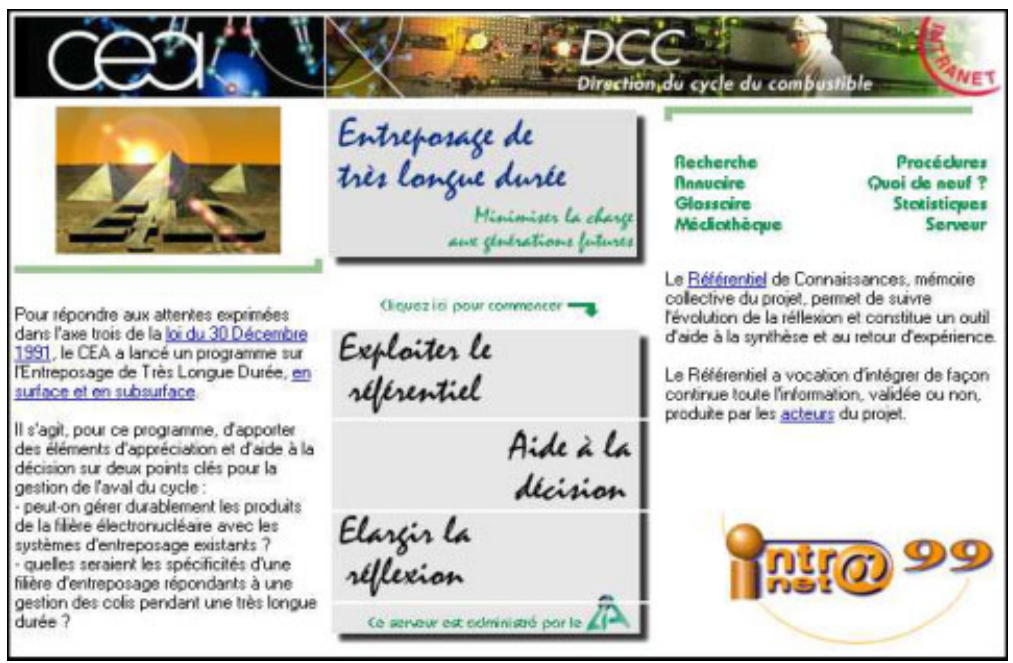

Figure 5. The home page of the RKB. 
studies were divided into sub-problems that were themselves structured into a hierarchy (Figure 6). In Figure 6, the structure of the document base is provided: each document is mapped in a grid where the columns are the potential solutions to be evaluated by the project and the rows are associated to the hierarchical breakdown of the problems into sub-problems.

Each EtLD document was mapped as proposed in the grid of Figure 6. However, because of the ever increasing number of documents, the next requirement of the EtLD manager was a tool integrated in the RKB to assess the progress of the concept evaluation and comparison processes: the basic idea was to monitor the scientific production of the project dimension by dimension in order to identify where there was a lack of documents revealing a malfunctioning or deficiency of studies in the project. Indeed, the head manager was aware that it was a complex and time-consuming task to periodically extract the essential results from a huge document base for the CNE. Initially simple statistics functionalities were implemented in the second version of the RKB: number of documents for each case of the grid, per day, month, project team and so on with a colour code to make the supervision activity easier from a man-machine viewpoint (Figure 7). It was supposed to indicate deficiency and redundancy in the studies.

However, the first reports to the CNE appeared more fastidious than expected. Indeed, a report to the CNE was not a mere summary of the numerous studies achieved in the EtLD framework but the scientific authorities were expecting an interpretation of these studies in terms of the objectives of the EtLD project. The head manager thus proposed to distinguish the scientific information and the knowledge useful to the project (the actionable knowledge) (Penalva and Mountmain 2002; Montmain et al., 2002). The notion of KI was introduced into the project.

The EtLD engineers were thus supposed to draft KIs as soon as they had analysed a document
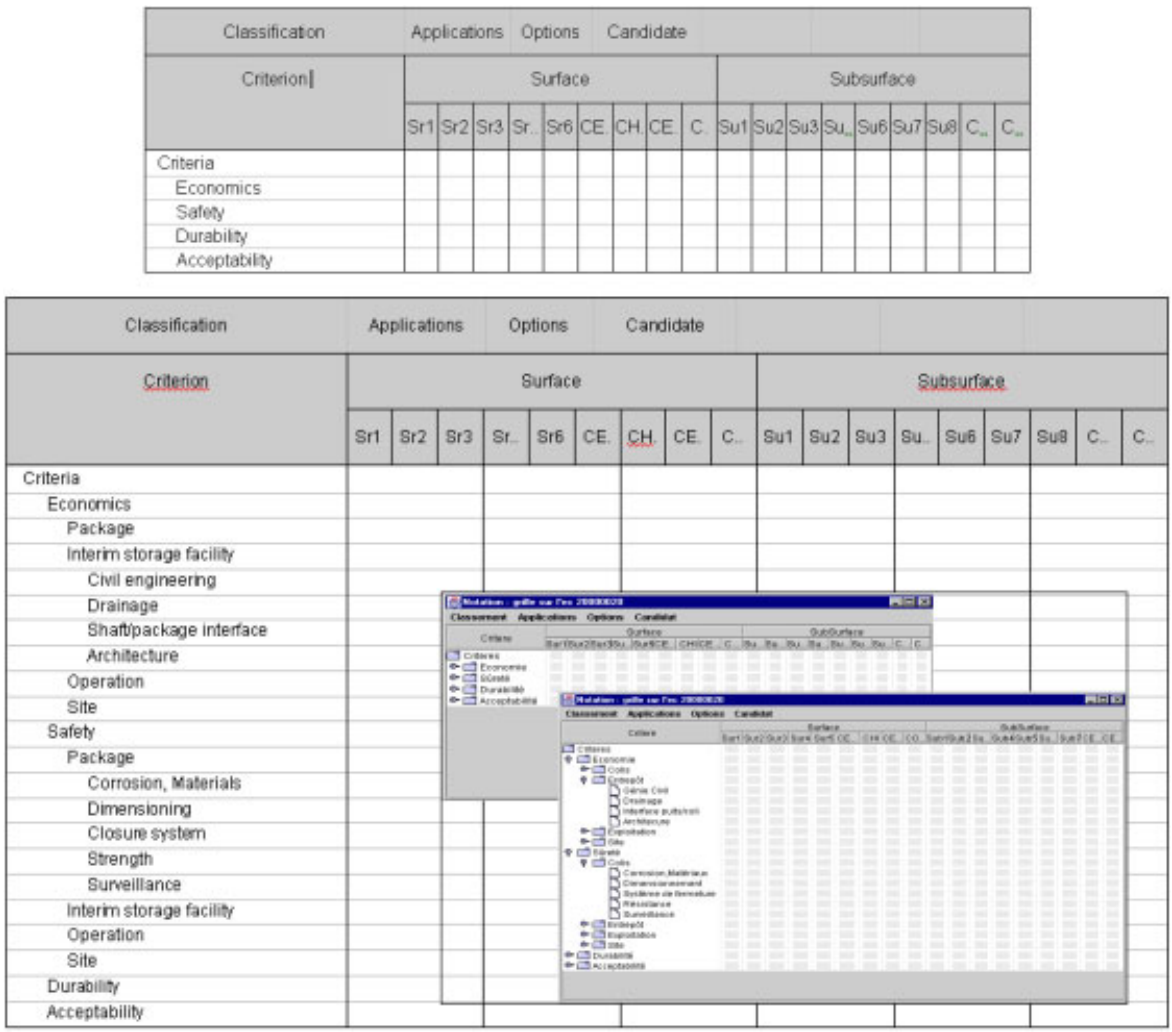

Figure 6. Hierarchical breakdown of the EtLD problematics and document map. 
(Figure 8): many documents, scientific or technical, research or engineering, technical or economical assessments were synthesized as electronic post-its that were finally managed at the RKB. The same map as for the documents was used for the KIs. Thus, reporting to the CNE became easier: the KIs were useful rhetorical elements structured into the EtLD problematic that could directly be used to answer the scientific and political authorities' questions. They constituted

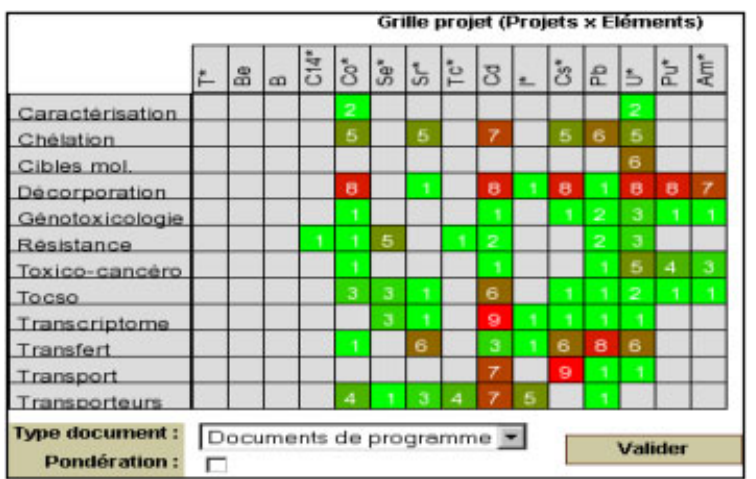

Figure 7. Statistics as observers of the scientific production of the project. technical, economical or scientific interpretations of documents in terms of EtLD objectives.

A last step remained: the exponential growth of the KIs made the automation of the rhetorical legitimating of the research indispensable. Because a KI was considered to be an interpretation of raw scientific, economical or engineering information, it constituted a value judgement by its writer with regard to the analysed piece of information (Figure 8). The EtLD head manager could thus consider using these value judgements mapped and managed at the RKB for monitoring purposes. The sub-problematics of the KIs map were derived into evaluation criteria of the project. Evaluation support functionalities were thus derived from a grid for score the possible solutions proposed over the life of the project, based on selected criteria. The KIs mapped on this grid and the value judgements they represented were then used to assess the solutions according to the considered criteria. The monitoring of the solution evaluation by the KIs provided a more efficient tool to the head manager to supervise the actionable knowledge of the project than the mere previous statistics indicators. The only difficulty was to convince the KIs' writers (the EtLD engineers) to convert value judgements in natural language into qualitative scores, the experience shows this had

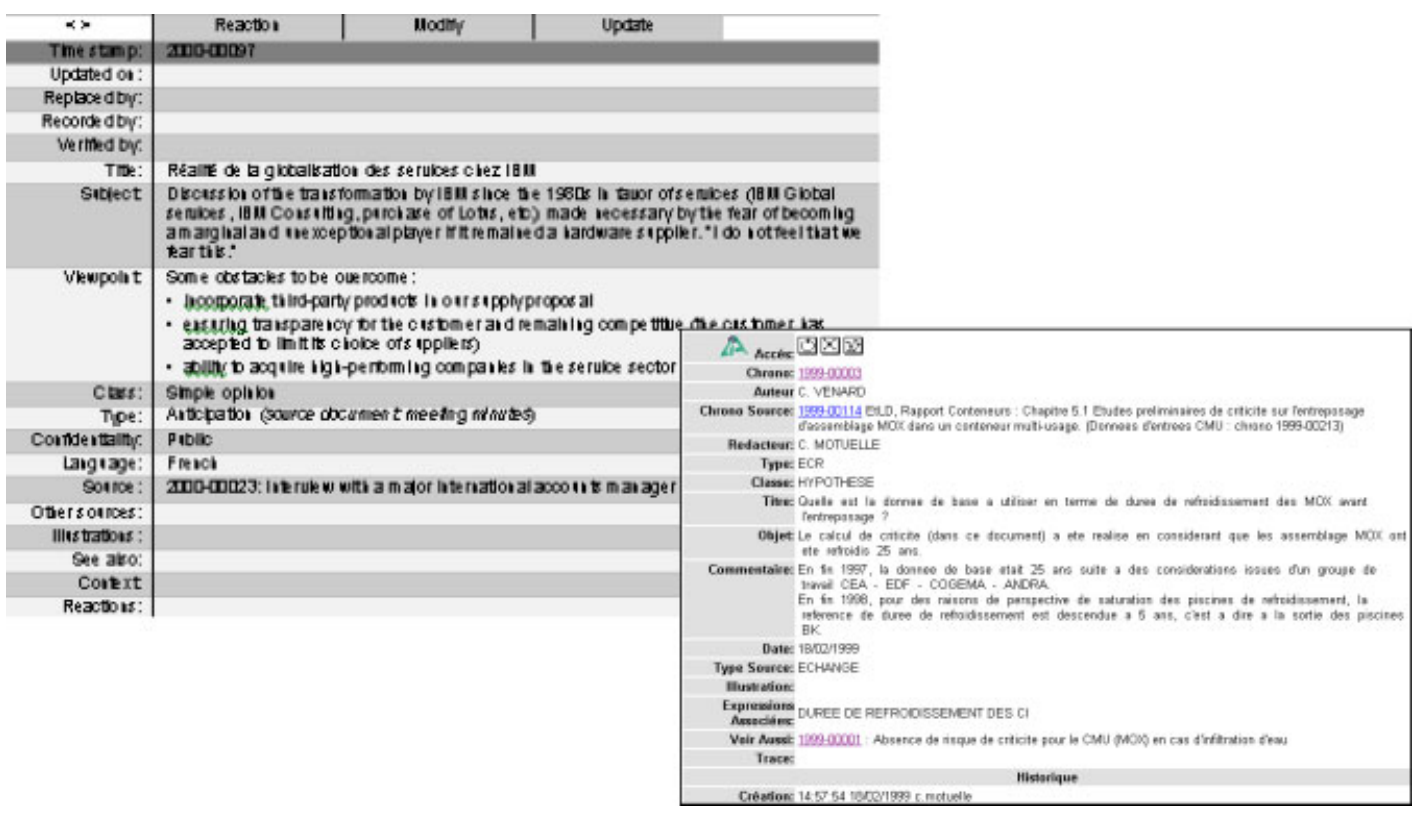

Figure 8. KI examples. 
been possible. Thanks to the KI's scores, the multicriteria evaluation of the competing solutions described in this paper could be implemented. The evaluation and elucidation functions could be achieved as proposed in Section 4.3 and facilitate the reports for the CNE: the system could partially support the automated argumentation of scientific, economical or technical facts that have appeared as remarkable decisional elements.

Finally, the EtLD head manager could directly use the risk evaluation and the recommendation algorithm to identify which dimensions in the evaluation could be considered as decidable or not, i.e. the supremacy of a solution w.r.t. a given evaluation criterion had been determined by a sufficient and relevant amount of KIs. This kind of indicator was extremely powerful to plan the future specific studies: the head manager knew exactly where studies were the most relevant to get a decidable answer to precise issues of the project. Our KDMS-DMSS coupling thus proposed a controlled dynamics of knowledge corpus that, in return, provided indications on the priority issues to be further studied in the project.

Document management activity is generally considered as an additional and time-consuming task by the project team in charge of the project. It is rather perceived as management keeping tabs on engineers' results! Thus, the evolution of the knowledge corpus related to a project does not necessarily reflect the project progress. Consequently, assigning such a goal to knowledge management probably is rewarding. All the engineers of the project become actors in the DMP.

\section{CONCLUSION}

From a general viewpoint, our approach deals with the place and impact of Information and Communication Sciences \& Technologies in collective and public technical and strategic choices: consensus building and DM in technical-social systems. This approach originates in cognitive considerations upon the DMP but proposes a mathematical and software instrumentation to implement the descriptive and cognitive DM model of Simon.

The KDMS is one element of an approach to compiling and using the documents produced within the context of a project. Integrating collective decision support functionalities-and more specifically evaluation and argumentation aids-confers an essential added value on the KDMS. Decision argumentation, effective use of expert documents from the KDMS in evaluating solutions, and the participation of a larger number of actors in the project evaluation process are invaluable tools and indicators for the project manager. Coupling a KDMS with a DMSS is natural: any decision must be based on the knowledge corpus managed by the KDMS.

The evaluation functionality of our DMSS relies on multi-criteria aggregation techniques. Characterizing conjunctive, disjunctive or compromise behaviour, modelling the relative importance of criteria, redundancy or synergy among the objectives involved in the decision process are at the heart of our work. Aggregation operators such as the Choquet integral in particular are capable of modelling interactions among objectives, and thus of avoiding the construction of an often overly constraining hierarchy of independent and additive objects.

The aggregation operator synthesizing the decision strategy is then submitted to a sensitivity analysis to select the most discriminating criteria in the final choice. Explanation relies on the notion of marginal contribution of a criterion. Pursuing this analysis for each of the relevant criteria then allows to extract the most decisive items of relevant knowledge from the KDMS. The justification of the decision strategy can thus be automated using only the KDMS base, allowing argumentation reports to be generated automatically.

The paper also proposes a way to capture the iterative cognitive phases-intelligence, design, choice and review-of Simon in a control theory framework. The cognitive loop of Simon's model is seen as a control loop on the decisional risk itself related to the entropy of the knowledge corpus. Our KDMS-DMSS coupling proposed a controlled dynamics of knowledge corpus that, in return, provided indications on the key issues to be further studied deepened in a project.

\section{REFERENCES}

Akharraz A, Mauris G, Montmain J. 2002. A project decision support system based on an elucidative fusion system. Fifth International Conference on Information Fusion, ICIF02, Annapolis, USA, 593-599. 
Akharraz A, Montmain J, Denguir A, Mauris G. 2004a. Information system and decisional risk control for a cybernetic modeling of project management. Modelling, Computation and Optimization in Information Systems and Management Sciences, Metz, France.

Akharraz A, Montmain J, Mauris G. 2004b. Elucidation and risk expressions of a movie recommendation based on a multi-criteria aggregation with a Choquet integral. IPMU'2004, Tenth International Conference on Information Processing and Management of Uncertainty in Knowledge-Based Systems, Genova, Italy.

Angilella S, Greco S, Lamantia F, Matarazzo B. 2004. Assessing non-additive utility for multicriteria decision aid. European Journal of Operational Research 158: 734-744.

Argyris C, Schön D. 1978. Organizational Learning: A Theory of Action Perspective. Addison-Wesley: Reading, MA.

Berrah L, Mauris G, Foulloy L, Haurat A. 2000. The Choquet integral as a tool for industrial performance aggregation. IPMU'2000, 8th International Conference on Information Processing and Management of Uncertainty in Knowledge-Based Systems, Madrid, Spain, 1937-1940.

Berrah L, Mauris G, Montmain J. 2005. Industrial performance improvement: efficacy and efficiency measurement issued from a Choquet integral aggregation. International Conference on Industrial Engineering and Systems Management (IESM 2005), Marrakech, Morocco.

Dasarathy BV. 2000. Elucidative fusion systems: an exposition. Information Fusion 1: 5-15.

Denguir-Rekik A, Mauris G, Montmain J. 2006. Propagation of uncertainty by the possibility theory in Choquet integral based decision making: application to an E-business website choice support. IEEE Transactions on Instrumentation and Measurement 55(33): 721-728.

Dubois D. 1983. Modèles mathématiques de l'imprécis et de l'incertain en vue d'applications aux techniques d'aide à la décision. Thèse de Docteur es-sciences, de l'Institut National Polytechnique de Grenoble.

Dubois D, Prade H. 1984. Criteria aggregation and ranking of alternatives in the framework of fuzzy set theory. In Fuzzy Sets and Decision Analysis, Zimmermann H-J, Zadeh LA, Gaines B (eds). TIMS Studies in the Management Sciences 20: 209-240. Elsevier: North Holland.

Dubois D, Prade H. 1985. A review of fuzzy set aggregation connectives. Information Sciences $\mathbf{3 6}$ : $85-121$.

Grabisch M. 1996. The application of fuzzy integrals in multicriteria decision making. European Journal of Operational Research 89: 445-456.

Grabisch M. 1997. k-ordered discrete fuzzy measures and their representation. Fuzzy Sets and Systems 92: 167-189.
Grabisch M, Nguyen HT, Walker EA. 1995. Fundamentals of Uncertainty Calculi, with Applications to Fuzzy Inference, Chapter 8. Kluwer Academic Publishers: Dordrecht.

Grabisch M, Orlowski SA, Yager RR. 1998. Fuzzy aggregation of numerical preferences. In Fuzzy Sets in Decision Analysis, Operations Research and Statistics, Slowinski R (ed.). Kluwer Academic Publishers: Dordrecht.

Grabisch M, Roubens M. 2000. Application of the Choquet integral in multicriteria decision making. In Fuzzy Measures and Integrals: Theory and Applications, Grabisch M, Murofushi T, Sugeno M (eds). Physica-Verlag: Wurzburg.

Kacprzyk J. 1987. Towards 'human-consistent' decision support systems through commonsense knowledgebased decision making and control models: a fuzzy approach. Computers and Artificial Intelligence 6(2): 97-122.

Koning J-L. 1990. Un mécanisme de gestion de règles de décision antagonistes pour les systèmes à base de connaissances. Thèse de l'Université Paul Sabatier de Toulouse.

Marichal J-L. 1998. Aggregation operators for mulitcriteria decision aid. Ph.D. Thesis, University of Liege.

Marichal JL, Roubens M. 2000. Determination of weights of interacting criteria from a reference set. European Journal of Operational Research 124(3): 641-650.

Montmain J, Akharraz A, Mauris G. 2002. Knowledge management as a support for collective decisionmaking and argumentation processes. IPMU'2002, Ninth International Conference on Information processing and Management of Uncertainty in KnowledgeBased Systems, Annecy, France.

Montmain J, Denguir A, Tardy J. 2004. An interactive decision-making support system for organization management and diagnosis. Modelling, Computation and Optimization in Information Systems and Management Sciences, Metz; France.

Moraïtis P, Tsoukiàs A. 2003. Decision aiding and argumentation. EUMAS 03 Workshop, Oxford, 1-12.

Niculae C, French S. 2003. Bringing understanding in societal decision making: explaining and communicating analyses? Journal of Mulit-Criteria Decision Analysis 12(2-3): 191-202.

Paschetta E, Tsoukiàs A. 2000. A real world MCDA application: evaluating software. Journal of MultiCriteria Decision Analysis 9: 205-226.

Penalva J-M, Montmain J. 2002. Travail collectif et intelligence collective: les référentiels de connaissances. IPMU'2002, 9th International Conference on Information Processing and Management of Uncertainty in Knowledge-Based Systems, Annecy, France.

Roy B. 1998. A missing Link in OR-DA, robustness analysis. Foundations of Computing and Decision Sciences 23(3):141-160.

Roy B. 2004. Pradigms and Challenges. in MCDA. In Multiple Criteria Decision Analysis, Figueira J, 
Greco S, Ehrgott M (eds). Kluwer Academic Publishers: Dordrecht, 3-24.

Roy B, Bouyssou D. 1993. Aide multicritère à la décision: méthodes et cas. Economica, Paris.

Sfez L. 1992. Critique de la décision. Presses de la Fondation des sciences politiques (4th edn) (1st edn, 1973).

Simon HA. 1991. Bounded rationality and organizational learning. Organization Science 2: 125-139.

Simon HA. 1997. Models of Bounded Rationality. MIT Press: Cambridge, MA.

Slowinski R. 1998. Fuzzy sets in decision analysis, operations research and statistics. The Handbook of Fuzzy Set. Kluwer Academic Publishers.
Vincke Ph. 1999a. Robust and neutral methods for aggregating preferences into an outranking relation. European Journal of Operational Research 112(2): 405-412.

Vincke Ph. 1999b. Robust solutions and methods in decision-aid. Journal of Multi-Criteria Decision Analysis 8(3): 181-187.

Yager R. 1988. On ordered weighted averaging aggregation operators in multicriteria decision-making. IEEE Transaction Systems, Man and Cybernetics 18: 183-190.

Zadeh L. 1983. A computational approach to fuzzy quantifiers in natural languages. Computers and Mathematics with Applications 9: 149-184. 